\title{
Standardised high dose versus low dose cranberry Proanthocyanidin extracts for the prevention of recurrent urinary tract infection in healthy women [PACCANN]: a double blind randomised controlled trial protocol
}

Babar Asma ${ }^{1,2} \mathbb{D}$, Leblanc Vicky², Dudonne Stephanie ${ }^{2}$, Desjardins Yves ${ }^{2}$, Howell Amy ${ }^{3}$ and Dodin Sylvie 1,2* $^{*}$

\begin{abstract}
Background: Urinary tract infections (UTIs) are amongst the most common bacterial infections affecting women. Although antibiotics are the treatment of choice for UTI, cranberry derived products have been used for many years to prevent UTIs, with limited evidence as to their efficacy. Our objective is to assess the efficacy of a cranberry extract capsule standardized in A-type linkage proanthocyanidins (PACS) for the prevention of recurrent urinary tract infection.

Methods: We will perform a 1:1 randomized, controlled, double blind clinical trial in women aged 18 years or more who present $\geq 2$ UTIs in 6 months or $\geq 3$ UTIs in 12 months. One hundred and forty-eight women will be recruited and randomized in two groups to either receive an optimal dose of cranberry extract quantified and standardized in PACs $(2 \times 18.5 \mathrm{mg}$ PACs per day) or a control dose $(2 \times 1 \mathrm{mg}$ PACs per day). The primary outcome for the trial is the mean number of new symptomatic UTIs in women during a 6-month intervention period. Secondary outcomes are: (1) To evaluate the mean number of new symptomatic UTIs with pyuria as demonstrated by a positive leucocyte esterase test; (2) To detect the mean number of new symptomatic culture-confirmed UTIs; (3) To quantify urinary PACs metabolites in women who take a daily dose of $37 \mathrm{mg}$ PACs per day compared to women who take a daily dose of 2 mg per day for 6 months; (4) To characterize women who present recurrent UTI based on known risk factors for recurrent UTI; (5) To describe the side effects of daily intake of cranberry extract containing $37 \mathrm{mg}$ PACs compared to 2 mg PACs. This report provides comprehensive methodological data for this randomized controlled trial.
\end{abstract}

Discussion: The results of this trial will inform urologists, gynaecologists, family physicians and other healthcare professionals caring for healthy women with recurrent UTI, as to the benefits of daily use of an optimal dose of cranberry extract for the prevention of recurrent UTI.

Trial registration: Clinicaltrials.gov, identifier: NCT02572895 October 8th 2015.

Keywords: Recurrent urinary tract infection, Women's health, Proanthocyanidins, Cranberry, Vaccinium macrocarpon, Antioxidants, Prevention

\footnotetext{
* Correspondence: sylvie.dodin@fmed.ulaval.ca

'Department of Obstetrics and Gynaecology, Laval University, CHU de

Québec - Université Laval, 2705, boulevard Laurier, Local A1385, Québec,

Québec G1V 4G2, Canada

${ }^{2}$ Institute of Nutrition and Functional Foods, Laval University, 2440 Hochelaga

Boulevard, Quebec City, Quebec G1V OA6, Canada

Full list of author information is available at the end of the article
}

(c) The Author(s). 2018 Open Access This article is distributed under the terms of the Creative Commons Attribution 4.0 International License (http://creativecommons.org/licenses/by/4.0/), which permits unrestricted use, distribution, and reproduction in any medium, provided you give appropriate credit to the original author(s) and the source, provide a link to the Creative Commons license, and indicate if changes were made. The Creative Commons Public Domain Dedication waiver (http://creativecommons.org/publicdomain/zero/1.0/) applies to the data made available in this article, unless otherwise stated. 


\section{Background}

Urinary tract infections (UTIs) are one of the most common bacterial infections affecting women [1, 2]. UTI preferentially affects young, sexually active women with $50-60 \%$ of women reporting at least one UTI during their lifetime [2]. Nearly 1 in 3 women will experience at least one episode of UTI requiring antibiotic therapy before the age of 24 years and a quarter of these women will present reoccurrence within 6 months [1] Anatomical differences in male and female perineal anatomy may explain why women are more susceptible than men to the ascension of faecal bacteria in the urinary tract. More precisely, these anatomical features include a relative shortness of the urethra [3], the urethral meatus's proximity to the anus and a more humid surrounding environment comparatively to the male anatomy [4, 5]. Additional well-established risk factors include spermicide-based contraception, history of previous UTI, first UTI before 15 years of age and those with UTI history in the mother [6-10].

Recurrent UTIs ( $r-U T I)$ are defined as more than 2 episodes in the last 6 months or 3 episodes in the last year [11]. The major symptoms of UTI include dysuria, increased frequency of urine, cloudy urine and occasionally hematuria [12]. In general, uncomplicated UTIs are confined to the bladder, resolve quickly following antibiotic treatment and thus are associated with fewer severe or long-term sequelae [13]. Though viewed as a benign affliction, uncomplicated lower UTI symptoms can have considerable impacts on the patient's productivity and quality of life. A study of university women reported that patients suffering from UTI experienced 2.4 days of restricted activity, 1.2 days of lost time and 0.4 bed bound days due to their symptoms [7]. Presently, the Canadian Urological Association and Society of Obstetricians and Gynecologists of Canada recommendations for the treatment of uncomplicated r-UTI use one of three antibiotic treatment regimens: continuous antibiotic prophylaxis, post-coital antibiotic prophylaxis or self-start antibiotic therapy [11, 12]. Empirical and preventive antibiotics for the treatment of r-UTI have been established as the most cost-effective way to manage these infections. However, prescribing without confirmation of diagnosis and isolation of causal bacterial contributes to the growing problem of uropathogen resistance in primary care [14].

Cranberries have been used for numerous years to prevent UTIs. Research suggests that proanthocyanidins (PACs), a component of cranberries, inhibit the adherence of p-fimbriated Escherichia coli on uroepithelial cells of the bladder, preventing the adherence of bacteria to the mucosal surface of the urinary tract and thereby inhibiting bacterial proliferation [15]. A multicentre randomized clinical trial (RCT) in sexually active adult women showed that a daily dose of $36 \mathrm{mg}$ PACs or more provided an optimal antibacterial effect in the urine [16]. A Cochrane systematic review published in 2012, could not definitively conclude on the efficacy of cranberry products for the prevention of r-UTI mainly because of a lack of observance to the intake of cranberry supplements in juice form and also because of varying PACs concentrations in the different clinical trials that were rarely quantified or standardized [17]. A systematic review in 2013 including trials conducted in women with r-UTI found a significantly decreased risk of UTI among women receiving cranberry-containing products compared to control ( 2 trials, Risk ratio $(\mathrm{RR})=0.53,95 \% \mathrm{CI}$ $0.33-0.83, \mathrm{I}^{2}=0 \%$ ) [18].

Since these reviews, several well-designed trials have evaluated the effects of daily cranberry capsules in women with r-UTI. Most notably, an RCT compared the preventive effects of a cranberry juice (UR65) containing $40 \mathrm{mg}$ PACs versus placebo on the relapse of UTI in women aged 20-79 years presenting acute complicated or uncomplicated cystitis with a history of r-UTI during a sixmonth follow-up [19] The authors found no significant difference in the relapse rates of UTI between groups (log-rank test, $p=0.4209$ ). Similarly, a double blind RCT evaluated the effects of a daily dose of $500 \mathrm{mg}$ of a commercial cranberry fruit powder (2 mg PACs) compared to a placebo in 176 women who experienced at least 2 symptomatic UTI in the twelve months preceding the study [20]. In this trial, the proportion of women who experienced greater than one UTI in the cranberry powder group was significantly lower than in the placebo group during the six-month follow-up period (10.8\% vs $25.8 \%$, $p$ $=0.04)$. Although well designed, these studies presented several methodological flaws such as the inclusion of women with complicated UTIs related to catheterization in the former as well as the use of a sub-optimal dose of cranberries in the latter.

We hypothesize that the efficacy of cranberry products for the prevention of R-UTI in women will be strongly increased by the usage of an optimal dose of cranberry extracts in capsule form (standardized to $37 \mathrm{mg}$ PACs per day) and by an adequate measure of participant observance.

\section{Methods}

\section{Study design and objectives}

To assess the effects of a standardized cranberry extract in sexually active healthy women who present $\mathrm{r}$-UTI, we will undertake a double blind, prospective RCT with 2 arms comparing the mean number of new UTIs during a 6-month period after consumption of a standardized cranberry extract containing $37 \mathrm{mg}$ PACs $(2 \times 18.5 \mathrm{mg}$ PACs per day) with a control dose of $2 \mathrm{mg}$ PACs $(2 \times$ $1 \mathrm{mg}$ PACs per day) in women presenting r-UTI. This protocol was developed in accordance with the Standard 
Protocol Items: Recommendations for Interventional Trials (SPIRIT) Statement. The SPIRIT figure is illustrated in Fig. 1.

The primary objective is to evaluate, in sexually active women who present r-UTIs, the effects of a standardized cranberry extract containing $37 \mathrm{mg}$ type-A linkage PACs per day, compared to a control dose of $2 \mathrm{mg}$ PACs per day during a 6-month period on the incidence rate of newly symptomatic UTIs during a 6-month follow-up period. Secondary objectives are: (1) To evaluate the mean number of new symptomatic UTIs with pyuria as demonstrated by a positive leucocyte esterase test; (2) To detect the mean number of new symptomatic culture-confirmed UTIs; (3) To quantify urinary PACs metabolites in women who take a daily dose of $37 \mathrm{mg}$ PACs per day compared to women who take a daily dose of $2 \mathrm{mg}$ per day for 6 months; (4) To characterize women who present $r$-UTI based on known risk factors such as spermicidal contraception use, frequency of

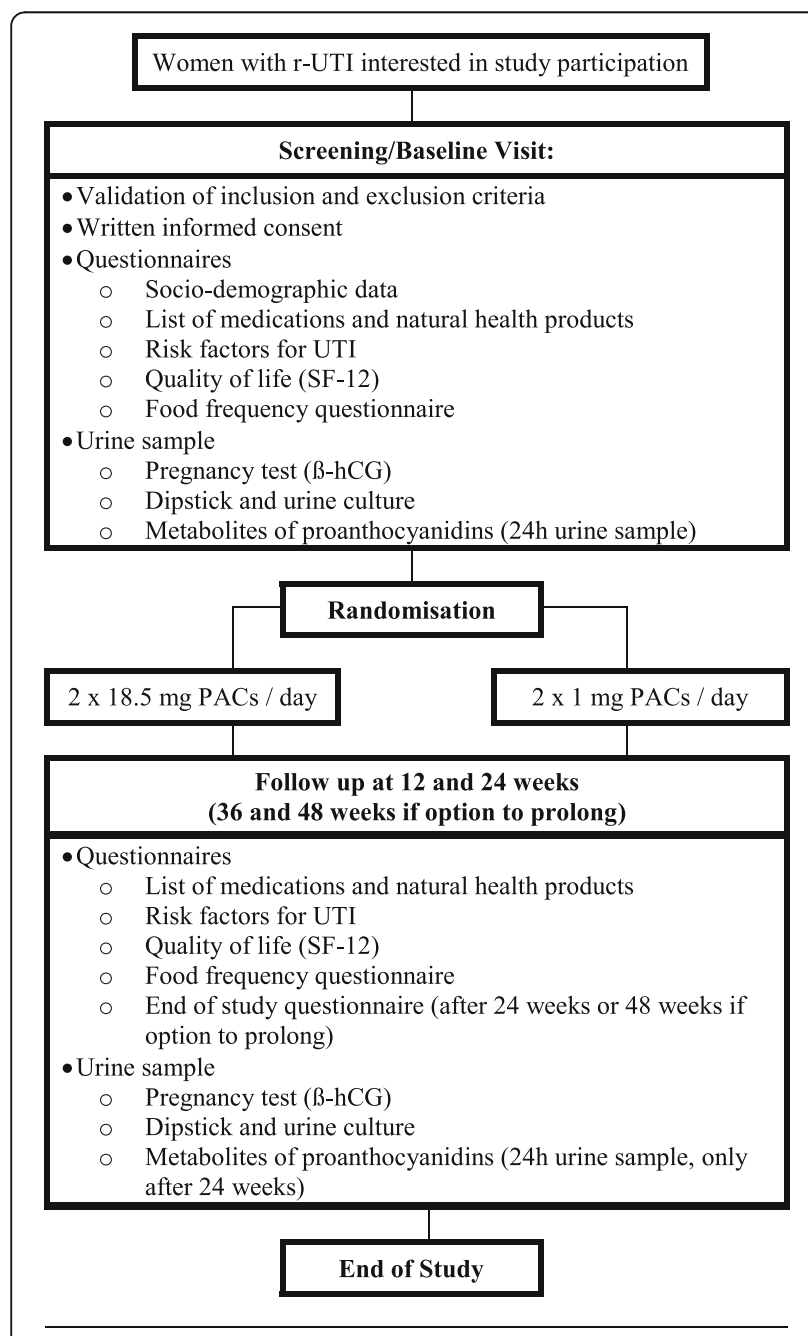

Fig. 1 Study procedures and characteristics of study visits sexual relations, personal and familial history of UTI; (5) To describe the side effects of daily intake of cranberry extract containing $37 \mathrm{mg}$ PACs compared to $2 \mathrm{mg}$ PACs.

\section{Study participants and recruitment}

This clinical trial aims to enrol sexually active nonpregnant non-lactating women aged 18 years and over presenting culture-confirmed $r$-UTI (defined as $\geq 2$ UTIs in the past 6 months or $\geq 3$ UTIs in the past 12 months). Women will be recruited in the Laval University community in Quebec City, Canada, through list serves and local clinician referrals as well as posters in medical clinics, social media, paid advertising and word of mouth. Women wishing to participate will contact the study coordinator who will explain the research project to them and verify eligibility according to inclusion and exclusion criteria (Table 1). The risks and benefits of the study will be thoroughly discussed and the consent form will be signed at the first of three visits at the Institute on Nutrition and Functional Foods (INAF).

Potential participants will need to restrain exposure to systemic antimicrobial agents or cranberry derivatives in the two weeks preceding enrolment. Women with anatomical abnormalities of the urinary tract, a history of renal disease (renal failure, nephrolithiasis) or intestinal disease causing malabsorption (Crohn's disease, Celiac disease), or anticoagulant therapy will be excluded. Furthermore, we will exclude women with known allergy or intolerance to cranberries.

\section{Study randomization}

Concealed randomization will be generated using computer aided block randomization by blocks of 10 . Eligible

Table 1 Admissibility criteria for the cranberry extract for prevention of recurrent urinary tract infections trial

Inclusion Criteria

Sexually active healthy women

Aged 18 years and older

Recent history of recurrent urinary tract infections $(U T I s)^{a}$

$\geq 2$ UTIs in the past 6 months and/or

$\geq 3$ UTIs in the past 12 months

No consumption of cranberry juice, polyphenol or antioxidant supplements in the last 2 weeks

Exclusion Criteria

Pregnancy

History of anatomical urogenital anomalies, urogenital tract surgery

History of acute or chronic renal failure, nephrolithiasis

History of intestinal diseases causing malabsorption

Anticoagulant medication in the last month

Known allergy or intolerance to cranberry

aUTIs diagnosed by a clinician and treated with antibiotic therapy 
women will be assigned 1:1 to consume a cranberry extract either formulated in high PAC content capsules (2 capsules of $18.5 \mathrm{mg}$ PAC per day) or low PAC content capsules (2 capsules of $1 \mathrm{mg}$ PAC per day) for 6 months. The low PAC content cranberry capsule is comparable to the majority of cranberry extract products presently approved by Health Canada [21] . The standardized capsules will be manufactured and provided by Diana Foods and will be distributed in opaque packaging in order to conceal colour variations from the research team. The PAC content of each cranberry treatment will be validated at INAF according to the standardized BL-DMAC method, using A2 procyanidin dimer as standard for the quantification [22]. All clinical investigation, laboratory analysis, data collection and assessment will be blinded to the randomization allocation.

\section{Clinical follow-up}

Each visit (0, 12 and 24 weeks) will include a short questionnaire documenting socio-demographic characteristics ( $T=0$ only), medication and natural health product intake, quality of life (SF-12) [23], risk factors for UTIs, and a validated food frequency questionnaire (FFQ) [24] modified for our study to specifically include foods containing PACs. Double data entry will be performed under the study coordinator supervision in order to promote data quality. Participants will be instructed to obtain a midstream urine sample according to standard methods suggested by the microbiological laboratory. A dipstick urinalysis using Chemstrip 9 (Roche Diagnostics USA) and a pregnancy test ( $(-\mathrm{hCG})$ will be completed. Three $10 \mathrm{ml}$ tubes will be placed in $\mathrm{a}-80{ }^{\circ} \mathrm{C}$ freezer for urinary PAC metabolites measurements by ultra-high performance liquid chromatography coupled with mass spectrometry, and the urine sample for culture will be refrigerated and transported to the microbiology laboratory at the CHU de Quebec - Universite Laval within $24 \mathrm{~h}$ after collection.

During their participation, the women will be asked to contact the study coordinator if they present symptoms of UTI in order to schedule a visit at INAF to confirm the diagnosis by urinalysis and urine culture and to receive an appropriate antibiotic prescription based on their medical history and allergies. Women unable to present themselves at INAF will be prescribed antibiotics waiving a confirmation of their UTI. Women wishing to discontinue the consumption of capsules will be asked to present themselves at the 3 and 6-month visit in order to complete intention to treat analysis. The participants will be asked not to consume other products containing cranberry derivatives for the duration of the study and to consume their cranberry extracts $2-4 \mathrm{~h}$ preceding each visit. Participants will also be asked to limit intense physical activity for $24 \mathrm{~h}$ preceding each visit. After 24 weeks of participation, women will be provided with the option of prolonging their participation for an additional 24 weeks, with follow-up visits at 36 and 48 weeks. This data will provide information on seasonal variations in the incidence of UTI.

\section{Sample size and statistical analysis}

Statistical analyses will be performed using SAS 9.2 (SAS Institute Inc., USA). All analyses will be based on the intention-to-treat principle before unmasking the treatment groups. The baseline characteristics of both groups will be compared using a Student's t test for continuous variables and a generalized linear model for nominal variables. The Poisson regression model, which allows us to adjust for participants that were lost to follow up, will be used to compare the incidence of UTI during the 6-month follow up period as well as side effects of the treatment. Subgroup analyses will be performed in order to evaluate the impact of cranberry capsules in pre- and post-menopausal women as well as women with certain risk factors for complicated UTI such as pelvic floor disorders and diabetes.

Based on the literature [17], we estimate that $35 \%$ of patients in the control group will present at least one UTI during the 6-month follow-up period. In total, 126 women will need to be recruited in order to detect a clinically significant difference of $25 \%$ between the 2 groups (10\% of women assigned to the experimental group will have at least 1 UTI with a power of $80 \%$ ). Based on our past clinical trial experience [25], we estimate that $15 \%$ of randomized participants will be lost to follow up, therefore 148 women will need to be recruited in order to have at least 126 participants who will complete the 24-week intervention.

\section{End points}

Incidence of UTI

The primary endpoint is the average number of symptomatic UTIs during the 6-month follow-up period. Individuals with acute urinary symptoms such as pollakiuria, urgency, burning, suprapubic pain, and hematuria will be assessed by study staff and will have to provide a urine sample for urinalysis. Women who present both symptom and pyuria criteria, defined as a positive leukocyte esterase dipstick result, will be diagnosed as having confirmed symptomatic UTI and prescribed appropriate antibiotic treatment. If the urine culture results are positive, the episode will be categorized as a culture-confirmed UTI. Women with symptomatic UTI during the study period will continue to take the cranberry capsules and remain in the study for the full 6 months unless they are lost to follow up or discontinue the intervention.

\section{Urinary PAC content}

At the beginning of the trial, participants will be offered the option to provide a 24-h urine collect for targeted metabolomic characterization of PAC metabolites using 
ultra-high-performance liquid chromatography coupled to tandem mass spectrometry, performed by a chemist blinded to treatment allocation.

\section{Compliance and side effects}

The cranberry extract capsules will be distributed at each visit and participants will be asked to bring remaining capsules the next visit in order to count remaining capsules. The participants will fill out a daily journal to record compliance, transient UTI symptoms and any adverse effects related to capsule intake. A bi-monthly email reminder will be sent to encourage participation. Each participant will receive an email reminder in the week preceding each visit. Evaluation of side effects will take place at each visit and participants will be asked to document symptoms (nausea, dyspepsia, abdominal pain, bloating and headaches) in their daily journal. In the presence of severe side effects, participants will be allowed to discontinue the intervention and remain in the study in order to preserve the intention to treat analyses.

\section{Blinding and contamination Bias}

The proportion of women who will guess their group allocation correctly will be documented with a short questionnaire at the last visit. To control for contamination bias, any antibiotic therapy during the study period will be declared to the study coordinator and PAC consumption will be measured by FFQ for the $24 \mathrm{~h}$ preceding each visit.

\section{Discussion}

Use of cranberry derived products in the prevention of r-UTI remains controversial, with no definitive evidence to show superiority of the cranberry compared to antibiotic therapy $[17,18]$. There is some evidence that cranberry products may reduce the incidence of UTIs compared to placebo, though the most effective amount and concentration of PACs that must be consumed and the duration for the intervention are unknown [17]. To our knowledge, this study is the first large, prospective randomized clinical trial assessing the impact of a cranberry extract capsule standardized to $37 \mathrm{mg}$ PAC per day compared to $2 \mathrm{mg}$ PAC per day in preventing UTIs in healthy women presenting $r$-UTI.

Incidence of symptomatic UTI during the 6-month follow-up period was selected as the primary end-point in this trial because it is the most important, clinically relevant long-term outcome for patients. We used three classifications for UTI analyses: symptomatic UTI, dipstick-positive UTI and culture-confirmed UTI. Symptomatic UTI was diagnosed if a participant presented at least one of the following symptoms: dysuria, pollakiuria, urinary urgency, suprapubic pain or hematuria. In the presence of clinical symptoms, participants will be asked to provide a urine sample in order to perform a dipstick test and a urine culture. Positive leucocytes or nitrites will indicate a positive dipstick test [26]. A positive urine culture will be designated by greater than $10^{6}$ colonyforming units, according to the microbiological laboratory standards of the CHUL hospital. In our study, antibiotics will be prescribed in the presence of clinical symptoms in combination with either a positive urine dipstick test and/or positive urine culture.

\section{Implications of findings}

Many Canadian women who present r-UTI commonly use over the counter cranberries-derived products with inadequate labelling of PACs concentrations. For cranberry products with quantification of PACs, these concentrations rarely exceed $2 \mathrm{mg}$ in Canada [21]. Various trials have tested the effectiveness of cranberry derived products, essentially in juice form, and their results remain discordant mainly due to the lack of standardization and low doses of PACs in tested products. Hence, the intrinsic activity of cranberry PACs, demonstrated against Escherichia coli in vitro, has never been optimized for the prevention of UTI. The results generated from this trial will clarify the role of cranberry extracts standardized in PACs on the decreased incidence of UTI in women presenting $r$-UTIs, will evaluate the differences in the incidence of UTI on the basis of different PACs concentrations and will respond to the recommendations reported in the last Cochrane meta-analysis [17].

This report provides comprehensive methods for a clinical trial on the prevention of RUTI by cranberry extract capsule intake. The strengths of this trial include the quantification and standardisation of PACs contained in the cranberry extract capsule and a randomized, double blind, controlled trial method. Our trial will add to a growing body of literature regarding cranberry extract capsule for the prevention of r-UTI in healthy women. In addition, the data set and specimen bank generated from conducting this trial will enable researchers to understand the metabolites of type-A PACs produced after prolonged consumption of cranberry capsules.

\section{Trial status}

Participant recruitment started on August 18th 2015 and was completed in March 2017. Study follow up visits will continue into Winter 2018.

\section{Abbreviations \\ FFQ: Food frequency questionnaire; PAC: Proanthocyanidin; RCT: Randomized controlled trial; r-UTI: Recurrent UTI; UTI: Urinary tract infection}

\section{Acknowledgements}

We thank Iseult Grenier-Ouellet and Marie-Pier Bernard-Genest for their assistance in the clinical follow-up of participants during this clinical trial at the Institute of Nutrition and Functional Foods, Laval University. The authors are very grateful to the laboratory and clinical staff and all participants in this study. 


\section{Funding}

This research project was funded by the Ministry of Agriculture, Fisheries and Food of Quebec and Nutra Canada (now part of Diana Food). The funders had no role in the design and conduct of this clinical trial; the collection, management, analysis, and interpretation of data; the preparation, review, or approval of the manuscript; and the decision to submit the manuscript for publication. Nutra Canada manufactured and donated the cranberry capsules used in this study.

\section{Availability of data and materials}

All data sets will be password protected and only available to project investigators. Data sets, cleaned and blinded of any identifying participant information, as well as the full protocol, will be available after the completion of the trial on request to the contacting author.

\section{Dissemination of results}

Trial results will be accessible to health professionals and the scientific community through articles published in peer-reviewed journals and presentations at local, national and international scientific conferences in the fields of gynaecology, urology, nutraceuticals and antioxidants. Results will also be disseminated to the general public through interviews on community radio and the publication of news articles.

\section{Authors' contributions}

$A B$ performed recruitment, clinical follow up, interpretation of data, statistical analyses and writing of the manuscript; VL. contributed to the design of this study, recruitment, clinical follow up, interpretation of data, statistical analyses and revision of the manuscript; S. Dudonne undertook supervision of the biomarker measures and review of the manuscript, $A D$ and YD contributed to the original concept and design of this study; S. Dodin contributed to the original concept and design of this study, undertook supervision of the clinical follow-up of participants, and wrote the study grant. All authors read and approved the final manuscript.

\section{Ethics approval and consent to participate}

The protocol and consent form of this study were reviewed and approved by the institutional ethics committee of Laval University with approval number 2015-091 A-5/ 03-11-2016. Further changes to the study protocol will require ethics approval from the institutional ethics committee. The study coordinator will obtain written informed consent from all study participants. Women will be able to withdraw from the study at any time during their participation. Data will be entered electronically and original study forms will be kept locked at the study site and maintained in storage for a period of 25 years after the completion of the study. This randomized clinical trial is registered in ClinicalTrials.gov, identifier: NCT02572895.

\section{Competing interests}

The authors report no conflicts of interest. The authors alone are responsible for the content and writing of the paper.

\section{Publisher's Note}

Springer Nature remains neutral with regard to jurisdictional claims in published maps and institutional affiliations.

\section{Author details}

${ }^{1}$ Department of Obstetrics and Gynaecology, Laval University, CHU de Québec - Université Laval, 2705, boulevard Laurier, Local A1385, Québec, Québec G1V 4G2, Canada. ${ }^{2}$ Institute of Nutrition and Functional Foods, Laval University, 2440 Hochelaga Boulevard, Quebec City, Quebec G1V 0A6, Canada. ${ }^{3}$ Rutgers University, 125A Lake Oswego Rd., Chatsworth, NJ 08019, USA.

Received: 16 June 2017 Accepted: 18 April 2018

Published online: 02 May 2018

\section{References}

1. Czaja CA, Hooton TM. Update on acute uncomplicated urinary tract infection in women. Postgrad Med. 2006;119(1):39-45.

2. Foxman B, Barlow R, D'Arcy H, Gillespie B, Sobel JD. Urinary tract infection: selfreported incidence and associated costs. Ann Epidemiol. 2000;10(8):509-15.

3. Hickling DR, Sun TT, Wu XR. Anatomy and physiology of the urinary tract: relation to host defense and microbial infection. Microbiol Spectr. 2015;3(4)
4. Yamamoto S, Tsukamoto T, Terai A, Kurazono H, Takeda Y, Yoshida O. Genetic evidence supporting the fecal-perineal-urethral hypothesis in cystitis caused by Escherichia coli. J Urol. 1997;157(3):1127-9.

5. Hooton TM. Pathogenesis of urinary tract infections: an update. antimicrobial chemotherapy. 2000;46(Suppl A):1-7.

6. Strom BL, Collins M, West SL, Kreisberg J, Weller S. Sexual activity, contraceptive use, and other risk factors for symptomatic and asymptomatic bacteriuria. A case-control study. Ann Intern Med. 1987;107(6):816-23.

7. Foxman B, Frerichs RR. Epidemiology of urinary tract infection: I. Diaphragm use and sexual intercourse. Am J Public Health. 1985;75(11):1308-13.

8. McDonald AM, Knight RC, Campbell MK, Entwistle VA, Grant AM, Cook JA, Elbourne DR, Francis D, Garcia J, Roberts I, et al. What influences recruitment to randomised controlled trials? A review of trials funded by two UK funding agencies. Trials. 2006;7:9.

9. Foxman B, Frerichs RR. Epidemiology of urinary tract infection: II. Diet, clothing, and urination habits. Am J Public Health. 1985;75(11):1314-7.

10. Scholes D, Hawn TR, Roberts PL, Li SS, Stapleton AE, Zhao LP, Stamm WE, Hooton TM. Family history and risk of recurrent cystitis and pyelonephritis in women. J Urol. 2010;184(2):564-9.

11. Epp A, Larochelle A, Lovatsis D, Walter JE, Easton W, Farrell SA, Girouard L, Gupta C, Harvey MA, Robert M, et al. Recurrent urinary tract infection. J Obstet Gynaecol Can. 2010;32(11):1082-101.

12. Dason S, Dason JT, Kapoor A. Guidelines for the diagnosis and management of recurrent urinary tract infection in women. Can Urol Assoc J. 2011;5(5):316-22.

13. Little P, Merriman R, Turner S, Rumsby K, Warner G, Lowes JA, Smith H, Hawke C, Leydon G, Mullee $M$, et al. Presentation, pattern, and natural course of severe symptoms, and role of antibiotics and antibiotic resistance among patients presenting with suspected uncomplicated urinary tract infection in primary care: observational study. BMJ (Clinical research ed). 2010;b5633:340.

14. Giesen LG, Cousins G, Dimitrov BD, van de Laar FA, Fahey T. Predicting acute uncomplicated urinary tract infection in women: a systematic review of the diagnostic accuracy of symptoms and signs. BMC Fam Pract. 2010;11:78.

15. Gupta K, Chou MY, Howell A, Wobbe C, Grady R, Stapleton AE. Cranberry products inhibit adherence of p-fimbriated Escherichia coli to primary cultured bladder and vaginal epithelial cells. J Urol. 2007;177(6):2357-60.

16. Howell AB, Botto H, Combescure C, Blanc-Potard AB, Gausa L, Matsumoto T, Tenke P, Sotto A, Lavigne JP. Dosage effect on uropathogenic Escherichia coli anti-adhesion activity in urine following consumption of cranberry powder standardized for proanthocyanidin content: a multicentric randomized double blind study. BMC Infect Dis. 2010;10:94.

17. Jepson RG, Williams G, Craig JC. Cranberries for preventing urinary tract infections. Cochrane Database Syst Rev. 2012;10:CD001321.

18. Wang CH, Fang CC, Chen NC, Liu SS, Yu PH, Wu TY, Chen WT, Lee CC, Chen SC. Cranberry-containing products for prevention of urinary tract infections in susceptible populations: a systematic review and metaanalysis of randomized controlled trials. Arch Intern Med. 2012;172(13):988-96.

19. Takahashi S, Hamasuna R, Yasuda M, Arakawa S, Tanaka K, Ishikawa K, Kiyota $H$, Hayami $H$, Yamamoto $S$, Kubo $T$, et al. A randomized clinical trial to evaluate the preventive effect of cranberry juice (UR65) for patients with recurrent urinary tract infection. J Infection Chemotherapy : official journal of the Japan Society of Chemotherapy. 2013;19(1):112-7.

20. Fromentin E, Vostalova J, Vidlar A, Galandakova A, Vrbkova J, Ulrichova J, Student V, Simanek V. A randomized, double-blind, placebo-controlled clinical trial to investigate the efficacy of cranberry fruit powder (Pacran) in the prevention of recurrent urinary tract infection in women. FASEB J. 2014;28

21. Licensed Natural Health Products Database [https://www.canada.ca/en/ health-canada/services/drugs-health-products/natural-non-prescription/ applications-submissions/product-licensing/licensed-natural-health-productsdatabase.html].

22. Prior RL, Fan E, Ji H, Howell A, Nio C, Payne MJ, Reed J. Multi-laboratory validation of a standard method for quantifying proanthocyanidins in cranberry powders. J Sci Food Agric. 2010;90(9):1473-8.

23. Jenkinson C, Layte $R$, Jenkinson D, Lawrence $K$, Petersen S, Paice C, Stradling J. A shorter form health survey: can the SF-12 replicate results from the SF36 in longitudinal studies? J Public Health Med. 1997;19(2):179-86.

24. Goulet J, Nadeau G, Lapointe A, Lamarche B, Lemieux S. Validity and reproducibility of an interviewer-administered food frequency questionnaire for healthy French-Canadian men and women. Nutr J. 2004;3:13. 
25. Mogollon JA, Bujold E, Lemieux S, Bourdages M, Blanchet C, Bazinet L, Couillard C, Noel M, Dodin S. Blood pressure and endothelial function in healthy, pregnant women after acute and daily consumption of flavanolrich chocolate: a pilot, randomized controlled trial. Nutr J. 2013;12:41.

26. Deville WL, Yzermans JC, van Duijn NP, Bezemer PD, van der Windt DA, Bouter LM. The urine dipstick test useful to rule out infections. A metaanalysis of the accuracy. BMC Urol. 2004;4:4.

Ready to submit your research? Choose BMC and benefit from:

- fast, convenient online submission

- thorough peer review by experienced researchers in your field

- rapid publication on acceptance

- support for research data, including large and complex data types

- gold Open Access which fosters wider collaboration and increased citations

- maximum visibility for your research: over $100 \mathrm{M}$ website views per year 